\title{
Semi-Classical Limit Theorems for Hartree-Fock Theory
}

\author{
Joseph G. Conlon * \\ Department of Mathematics, University of Missouri, Columbia, MO 65211, USA
}

\begin{abstract}
Consider a large number of electrons with Coulomb repulsion moving under the influence of static nuclei. It is assumed the potentials due to the nuclei are Coulombic away from their centers but are smooth at the centers, so no singularities exist. The author shows that the exchange energy for the Hartree-Fock ground state of this system converges in a suitable limit to the formula obtained by Dirac for exchange energy as an integral of the one body density.
\end{abstract}

\section{Introduction}

In this paper we prove some semi-classical limit theorems for the Hartree-Fock theory associated with a Coulombic Hamiltonian of electrons interacting with static nuclei. We assume there are $k$ nuclei with positive charges $z_{j}, 1 \leqq j \leqq k$, situated at the points $R_{j} \in \mathbb{R}^{3}, 1 \leqq j \leqq k$, respectively. Let $g: \mathbb{R}^{3} \rightarrow \mathbb{R}$ be a continuous nonnegative spherically symmetric function with compact support whose integral over $\mathbb{R}^{\mathbf{3}}$ is 1 . We assume the electrostatic potential at $x \in \mathbb{R}^{\mathbf{3}}$ due to the nuclei is $-V(x)$, where

$$
V(x)=\sum_{j=1}^{k} z_{j} \int_{\mathbb{R}^{3}} \frac{g\left(y-R_{j}\right)}{|x-y|} d y .
$$

Thus for large $x$ the potential $-V(x)$ is the same as the Coulombic potential due to the $k$ nuclei, but for $x$ close to the points $R_{j}, 1 \leqq j \leqq k, V(x)$ is smoothed. Ideally we would like to assume simply that $V(x)$ is the Coulomb potential due to the $k$ nuclei. However the techniques of this paper do not apply to that case.

Next we introduce $n$ electrons, each with charge -1 and mass $m$, moving in the field of the potential $-V(x)$. Let $x_{i} \in \mathbb{R}^{3} \times\{-1,1\}=\Omega$ be the coordinate of the $i^{\text {th }}$ electron in the product of $\mathbb{R}^{3}$ with the spin space $\{-1,1\}$. Then the $n$ electron wave function $\psi$ may be written as $\psi \equiv \psi\left(x_{1}, \ldots, x_{n}\right)$, where $\psi \in L^{2}\left(\Omega^{n}\right)$. Let $\mathscr{H}_{n}$ be the

* $\quad$ Research supported by NSF Grant No. MCS 8100761 
Hilbert space of all such $\psi$ which are anti-symmetric in the $x_{i}, 1 \leqq i \leqq n$. By the Pauli exclusion principle $\mathscr{H}_{n}$ is the state space for the $n$ electron system. The corresponding Hamiltonian $H_{n}(V)$ is given by

$$
H_{n}(V)=-h^{2}\left(8 \pi^{2} m\right)^{-1} \sum_{i=1}^{n} \Delta_{i}-\sum_{i=1}^{n} V\left(x_{i}\right)+\sum_{i<j=1}^{n}\left|x_{i}-x_{j}\right|^{-1},
$$

with $h$ being Planck's constant, and $\Delta_{i}$ the Laplacian in the $x_{i}$ variable $1 \leqq i \leqq n$.

Here we are concerned with estimating asymptotically the ground state energy $E_{n}(V)$ of the $n$ electron system as $n \rightarrow \infty$. If $[$,$] denotes the inner product on \mathscr{H}_{n}$, then the Rayleigh-Ritz procedure yields

$$
E_{n}(V)=\inf \left\{\left[\psi, H_{n}(V) \psi\right]: \psi \in \mathscr{H}_{n},\|\psi\|=1\right\} .
$$

Let $z$ be defined by

$$
z=\sum_{j=1}^{k} z_{j}
$$

It is known [14] that if $n \leqq z$ there is a function $\psi \in \mathscr{H}_{n}$ which achieves the infimum in (1.2). This function is not necessarily unique.

In Hartree-Fock (HF) theory one takes the infimum in (1.3) over functions $\psi$ which are anti-symmetric products of single electron wave functions. Let $\psi_{1}(x), \ldots, \psi_{n}(x)$ be an orthonormal set in $L^{2}(\Omega)$ representing the wave functions of the $n$ electrons and $\psi$ the Slater determinant of the $\psi_{1}, \ldots, \psi_{n}$. Then

$$
\left[\psi, H_{n}(V) \psi\right]=\varepsilon_{\mathrm{HF}}\left(\psi_{1}, \ldots, \psi_{n}\right),
$$

where

$$
\varepsilon_{\mathrm{HF}}=K+\mathrm{A}+\mathrm{R}+\mathrm{Ex} .
$$

The kinetic energy $K$ is given by

$$
K\left(\psi_{1}, \ldots, \psi_{n}\right)=h^{2}\left(8 \pi^{2} m\right)^{-1} \sum_{i=1}^{n} \int_{\Omega}\left|\nabla \psi_{i}(x)\right|^{2} d x .
$$

The attractive and repulsive potential energies $A$ and $R$ may be expressed as integrals of the one body density

$$
\varrho(x)=\sum_{i=1}^{n}\left|\psi_{i}(x)\right|^{2}, \quad x \in \Omega
$$

Thus we have

$$
\begin{aligned}
\mathrm{A}(\varrho, V) & =-\int_{\Omega} \varrho(x) V(x) d x \\
\mathrm{R}(\varrho) & =\frac{1}{2} \int_{\Omega} \int_{\Omega} \frac{\varrho(x) \varrho(y)}{|x-y|} d x d y .
\end{aligned}
$$

The nonclassical exchange energy Ex is given by

$$
\operatorname{Ex}\left(\psi_{1}, \ldots, \psi_{n}\right)=-\frac{1}{2} \iint_{\Omega} \frac{\left|\sum_{i=1}^{n} \psi_{i}(x) \overline{\psi_{i}(y)}\right|^{2}}{|x-y|} d x d y
$$


Let $E_{n}^{\mathrm{HF}}(V)$ be the Hartree-Fock ground state energy for the system (1.2). Lieb and Simon [11] showed that if $n<z+1$, this ground state energy is achieved for some orthonormal set of single electron wave functions $\psi_{1}(x), \ldots, \psi_{n}(x)$. Let $\varrho(x)$ be the one body density (1.8) associated with these single electron wave functions. The net electrostatic potential at a point $x \in \mathbb{R}^{3}$ due to nuclei and electrons is $-\phi_{\varrho}(x)$, where

$$
\phi_{\varrho}(x)=V(x)-\int_{\Omega} \frac{\varrho(y)}{|x-y|} d y .
$$

The potential (1.12) is a local potential. We define a nonlocal potential operator $L\left(\psi_{1}, \ldots, \psi_{n}\right)$ by

$$
L\left(\psi_{1}, \ldots, \psi_{n}\right) u(x)=\sum_{i=1}^{n} \psi_{i}(x) \int_{\Omega} \frac{\overline{\psi_{i}(y)} u(y)}{|x-y|} d y,
$$

for $u \in L^{2}(\Omega)$. The Euler equation condition for the $\psi_{1}, \ldots, \psi_{n}$ is then that these functions are the first $n$ eigenfunctions of the operator

$$
S\left(\psi_{1}, \ldots, \psi_{n}\right)=-h^{2}\left(8 \pi^{2} m\right)^{-1} \Delta-\phi_{\varrho}(x)-L\left(\psi_{1}, \ldots, \psi_{n}\right) \text { on } L^{2}(\Omega) .
$$

Next we consider Thomas-Fermi theory. Suppose there is a system of $\lambda$ electrons moving under the potential $-V(x)$ with one body density $f(x), x \in \mathbb{R}^{3}$. Thus $f(x) \geqq 0, x \in \mathbb{R}^{3}$, and

$$
\int_{\mathbb{R}^{3}} f(x) d x=\lambda .
$$

The Thomas-Fermi (TF) kinetic energy of the system is

$$
K_{\mathrm{TF}}(f)=\frac{3}{5} c \int_{\mathbb{R}^{3}} f(x)^{5 / 3} d x,
$$

where the constant $c$ is given by

$$
c=h^{2}(2 m)^{-1} 3^{2 / 3}(8 \pi)^{-2 / 3} .
$$

The attractive and repulsive Thomas-Fermi potential energies are $\mathrm{A}(f, V)$ and $\mathrm{R}(f)$ respectively as in (1.9) and (1.10). Hence the Thomas-Fermi energy functional $\varepsilon_{\mathrm{TF}}(f, V)$ is given by

$$
\varepsilon_{\mathrm{TF}}(f, V)=K_{\mathrm{TF}}(f)+\mathrm{A}(f, V)+\mathrm{R}(f) .
$$

The Thomas-Fermi ground state energy for the $\lambda$ electron system, $E_{\dot{\lambda}}^{\mathrm{TF}}(V)$, is then obtained by minimizing (1.18) subject to the condition (1.15). Lieb and Simon [12] proved that if $\lambda$ satisfies the inequalities

$$
0<\lambda \leqq z
$$

there is a unique function $\varrho_{\lambda}^{\mathrm{TF}}(x) \in L^{1}\left(\mathbb{R}^{3}\right) \cap L^{5 / 3}\left(\mathbb{R}^{3}\right)$ which achieves this minimum. The corresponding potential function $\phi_{\lambda}^{\mathbf{T F}}(x)$ is defined by (1.12) with $\varrho=\varrho_{\lambda}^{\mathbf{T F}}$. The Euler equation for the variational problem is then

$$
c Q_{\lambda}^{\mathrm{TF}}(x)^{2 / 3}=\max \left[\phi_{\lambda}^{\mathrm{TF}}(x)-\phi_{\lambda}, 0\right], \quad x \in \mathbb{R}^{3},
$$

where the Lagrange multiplier $\phi_{\lambda}$ satisfies $\phi_{\lambda} \geqq 0$ and $\phi_{\lambda}=0$ if and only if $\lambda=z$. 
We relate Hartree-Fock theory to Thomas-Fermi theory. For $\alpha>0$ let $V_{\alpha}(x)$ be defined in terms of the potential (1.1) by

$$
V_{\alpha}(x)=\alpha^{4 / 3} V\left(\alpha^{1 / 3} x\right) \text {. }
$$

Letting $\lambda$ satisfy (1.19) we see that for any $\alpha>0$ such that $\alpha \lambda$ is an integer, the Hamiltonian $H_{\alpha \lambda}\left(V_{\alpha}\right)$ of (1.2) has a Hartree-Fock ground state which may be described by an orthonormal set of $\alpha \lambda$ wave functions in $L^{2}(\Omega),{ }_{\alpha} \psi_{1}, \ldots,{ }_{\alpha} \psi_{\alpha \lambda}$. Then if we let $\alpha \rightarrow \infty$ through integer values of $\alpha \lambda$ it is known [12] that

$$
\lim _{\alpha \rightarrow \infty} \alpha^{-7 / 3} E_{\alpha \lambda}^{\mathrm{HF}}\left(V_{\alpha}\right)=E_{\lambda}^{\mathrm{TF}}(V) .
$$

This result is proved by the method of Dirichlet-Neumann bracketing. By applying the Feynman-Hellman technique to (1.22) one can also see [12] that

$$
\lim _{\alpha \rightarrow \infty} \alpha^{-7 / 3} K\left({ }_{\alpha} \psi_{1}, \ldots,{ }_{\alpha} \psi_{\alpha \lambda}\right)=K_{\mathrm{TF}}\left(\varrho_{\lambda}^{\mathrm{TF}}\right)
$$

If we let $\varrho_{\alpha}(x)$ be the one body density (1.8) associated with the wave functions ${ }_{\alpha} \psi_{1}, \ldots,{ }_{\alpha} \psi_{\alpha \lambda}$, it follows similarly that

$$
\begin{gathered}
\lim _{\alpha \rightarrow \infty} \alpha^{-7 / 3} \mathrm{~A}\left(\varrho_{\alpha}, V_{\alpha}\right)=\mathrm{A}\left(\varrho_{\lambda}^{\mathrm{TF}}, V\right), \\
\lim _{\alpha \rightarrow \infty} \alpha^{-7 / 3} \mathrm{R}\left(\varrho_{\alpha}\right)=\mathrm{R}\left(\varrho_{\lambda}^{\mathrm{TF}}\right) .
\end{gathered}
$$

In 1930 Dirac obtained a semi-classical value for the exchange energy associated with a one body density $f(x)$ of electrons. His value is [4],

$$
\operatorname{Ex}_{D}(f)=-3^{4 / 3} \pi^{-1 / 3} 4^{-1} \int_{\mathbb{R}^{3}} f(x)^{4 / 3} d x .
$$

The main result of this paper is to relate the Hartree-Fock exchange energy to the Dirac exchange energy as follows:

$$
\lim _{\alpha \rightarrow \infty} \alpha^{-5 / 3} \operatorname{Ex}\left({ }_{\alpha} \psi_{1}, \ldots,{ }_{\alpha} \psi_{\alpha \lambda}\right)=\operatorname{Ex}_{D}\left(\varrho_{\lambda}^{\mathrm{TF}}\right)
$$

We also strengthen some results of Lieb and Simon [12] on the convergence of the Hartree-Fock one body density to the Thomas-Fermi one body density. We prove that for $x \in \mathbb{R}^{3}$,

$$
\lim _{\alpha \rightarrow \infty} \alpha^{-2} \varrho_{\alpha}\left(\alpha^{-1 / 3} x\right)=\varrho_{\lambda}^{\mathrm{TF}}(x)
$$

In order to prove (1.27) we use different techniques than those employed in [12]. The reason is that exchange energy is a lower order term in the Hartree-Fock energy functional. We need two results from the Lieb-Simon work. The first one is (1.23). The second, although not explicitly stated in their work, can be obtained by applying the Feynman-Hellman technique to (1.22). It is the following:

$$
\lim _{\alpha \rightarrow \infty}\left[\alpha^{-4 / 3} \phi_{\varrho_{\alpha}}\left(\alpha^{-1 / 3} x\right)-V(x)\right]=\phi_{\lambda}^{\mathrm{TF}}(x)-V(x), \quad x \in \mathbb{R}^{3},
$$

where $\phi_{\varrho_{\alpha}}(x)$ is the potential (1.12) associated with the electron density $\varrho_{\alpha}(x)$ and the nuclear potential $V_{\alpha}(x)$. 
This work stems from a previous paper [2], where we proved a version of (1.27). In Sect. 2 we obtain some Sobolev inequalities necessary for our work. In Sect. 3 we obtain (1.27) for the simple case of a large number of fermions with no mutual interaction moving under the influence of a nonlocal potential. Then in Sect. 4 we extend the method to the case under consideration.

For a review of the most recent rigorous work on Thomas-Fermi and related theories see [8].

\section{Sobolev Inequalities}

Let $L^{p}(\Omega)$ and $L^{p}\left(\mathbb{R}^{3}\right), 1 \leqq p \leqq \infty$, be respectively the spaces of functions on $\Omega$ and $\mathbb{R}^{3}$ whose $p^{\text {th }}$ powers are integrable. If a function $u(x)$ lies in one of these spaces, we denote its norm by $\|u\|_{p}$. We define the bilinear form (,) on functions $u, v$ with domains $\Omega$ or $\mathbb{R}^{3}$ such that $|u v|$ is integrable by

$$
(u, v)=\int \overline{u(x)} v(x) d x,
$$

where integration is over the appropriate domain, $\Omega$ or $\mathbb{R}^{3}$.

For functions $\psi \in L^{2}\left(\mathbb{R}^{3}\right)$ we associate an operator $L_{\psi}$ by

$$
L_{\psi} u(x)=\psi(x) \int_{\mathbb{R}^{3}} \frac{\overline{\psi(y)} u(y)}{|x-y|} d y .
$$

Lemma 2.1. (a) Let $r, s$ be such that $2<r, s<6$ and

$$
\frac{1}{r}+\frac{1}{s}=\frac{2}{3} \text {. }
$$

Then for $u \in L^{r}\left(\mathbb{R}^{3}\right), v \in L^{s}\left(\mathbb{R}^{3}\right)$, the inequality

$$
\left|\left(v, L_{\psi} u\right)\right| \leqq C_{r}\|\psi\|_{2}^{2}\|u\|_{r}\|v\|_{s}
$$

holds, where $C_{r}$ is a constant depending only on $r$.

(b) The operator $L_{\psi}$ is positive definite and there is a constant $C$ such that for $u \in H^{1}\left(\mathbb{R}^{3}\right)$,

$$
0 \leqq\left(u, L_{\psi} u\right) \leqq C\|\psi\|_{2}^{2}\|u\|_{2}\|\nabla u\|_{2} .
$$

Proof. (a) We use the Hardy-Littlewood-Sobolev inequality [13]. Thus if $p, q$ are such that $1<p, q<3 / 2$, and $p^{-1}+q^{-1}=5 / 3$, it follows from this inequality that

$$
\left|\left(v, L_{\psi} u\right)\right| \leqq C_{p}\|u \psi\|_{p}\|v \psi\|_{q},
$$

where the constant $C_{p}$ depends only on $p$. The result (2.4) follows by applying Hölder's inequality to the right side of (2.6) with

$$
r=\frac{2 p}{2-p}, \quad s=\frac{2 q}{2-q} .
$$

(b) The operator $L_{\psi}$ is positive definite since the function $|x|^{-1}$ is positive definite. The inequality (2.5) follows from (2.4) by putting $u=v, r=s=1 / 3$, and applying a Sobolev inequality [13]. 
Next let $\psi_{i}(x), i=1,2, \ldots$, be an orthonormal set in $L^{2}(\Omega)$. For each $N=1,2, \ldots$, we define an operator $L_{N}$ by

$$
L_{N} u(x)=\sum_{i=1}^{N} \psi_{i}(x) \int_{\Omega} \frac{\overline{\psi_{i}(y)} u(y)}{|x-y|} d y .
$$

Lemma 2.2. (a) Let $r$, s satisfy the conditions of Lemma 2.1. There is a $C_{r}$ depending only on $r$ such that for $u \in L^{r}(\Omega), v \in L^{s}(\Omega)$,

$$
\left|\left(v, L_{N} u\right)\right| \leqq C_{r} N^{1 / 2}\|u\|_{r}\|v\|_{s} .
$$

(b) The operator $L_{N}$ is positive definite and there is a constant $C$ such that for $u \in H^{1}(\Omega)$,

$$
0 \leqq\left(u, L_{N} u\right) \leqq C N^{1 / 2}\|u\|_{2}\|\nabla u\|_{2} .
$$

Proof. (a) Let us regard $u \in L^{r}(\Omega)$ and $v \in L^{s}(\Omega)$ as fixed functions. We define an operator $T$ on $L^{2}(\Omega) \cdot$ by

$$
T \psi(x)=v(x) \int_{\Omega} \frac{\overline{u(y)} \psi(y)}{|x-y|} d y .
$$

From the Hardy-Littlewood-Sobolev theorem one can see that $T$ is a bounded operator on $L^{2}(\Omega)$. Furthermore, we have

$$
\left|\left(v, L_{N} u\right)\right| \leqq \sum_{i=1}^{N}\left|\left(\psi_{i}, T \psi_{i}\right)\right| .
$$

The operator $T$ is Hilbert-Schmidt if

$$
\operatorname{Trace}\left(T T^{*}\right)=\int_{\Omega} \int_{\Omega} \frac{|v(x)|^{2}|u(y)|^{2}}{|x-y|^{2}} d x d y
$$

is finite. Applying the Hardy-Littlewood-Sobolev theorem to the integral in (2.13), we see that there is a constant $C_{r}$ depending only on $r$ such that

$$
\operatorname{Trace}\left(T T^{*}\right) \leqq C_{r}^{2}\|u\|_{r}^{2}\|v\|_{s}^{2} .
$$

The inequality (2.9) then follows from (2.12) and the fact that

$$
\begin{aligned}
\sum_{i=1}^{N}\left|\left(\psi_{i}, T \psi_{i}\right)\right| & \leqq N^{1 / 2}\left\{\sum_{i=1}^{N}\left|\left(\psi_{i}, T \psi_{i}\right)\right|^{2}\right\}^{1 / 2} \\
& \leqq N^{1 / 2}\left\{\operatorname{Trace}\left(T T^{*}\right)\right\}^{1 / 2}
\end{aligned}
$$

(b) Just as in Lemma 2.1 we take $u=v$ and $r=s=1 / 3$ in (2.9).

Remark. Lieb [9] has shown by using a result of Cwickel [3] that one can replace the constant $C_{r} N^{1 / 2}$ in (2.9) by $C_{r}^{1} N^{1 / 3}$, where $C_{r}^{1}$ depends only on $r$. It is easy to see that $N^{1 / 3}$ is the lowest power of $N$ which can occur in the inequality (2.9).

Finally we state a theorem of Lieb $[7,10]$ which shows that Dirac exchange energy is a lower bound for Hartree-Fock exchange energy. 
Lemma 2.3. Let $\psi_{1}(x), \ldots, \psi_{N}(x)$ be an orthonormal set in $L^{2}(\Omega)$ such that $\psi_{i}$ is in $H^{1}(\Omega), 1 \leqq i \leqq N$. Let $\varrho(x)$ be the one body density

$$
\varrho(x)=\sum_{i=1}^{N}\left|\psi_{i}(x)\right|^{2}, \quad x \in \Omega .
$$

Then for any Borel set $S \subset \Omega$, the following inequality holds:

$$
\frac{1}{2} \int_{S} \int_{S} \frac{\left.\sum_{i=1}^{N} \psi_{i}(x) \overline{\psi_{i}(y)}\right|^{2}}{|x-y|} d x d y \leqq C \int_{S} \varrho(x)^{4 / 3} d x,
$$

where $C$ is a universal constant.

Lemma 2.3 is Lieb's theorem stated for a Hartree-Fock wave function. Note that his theorem is stated for $S=\Omega$ but the argument extends to any $S \subset \Omega$.

\section{Semi-Classical Theorems for Fermions Moving Under a Nonlocal Potential}

We consider the operator $H=-\frac{1}{2} \Delta$ acting on $L^{2}\left(\mathbb{R}^{3}\right)$. It is well known that $H$ has a unique self-adjoint extension which we also denote by $H$. This self-adjoint operator generates a strongly continuous semi-group $H_{t}=\exp (-H t), t>0$, of integral operators $H_{t}$ with kernels

$$
H_{t}(x, y)=(2 \pi t)^{-3 / 2} \exp \left[-|x-y|^{2} / 2 t\right], \quad t>0 .
$$

Next let $W(x)$ be a continuous potential in $L^{\infty}\left(\mathbb{R}^{3}\right)$. The operator $T=H+W$ is then essentially self-adjoint on $L^{2}\left(\mathbb{R}^{3}\right)$ and generates a strongly continuous semigroup $T(t)=\exp (-T t), t>0$, of integral operators [13]. The kernel of the operator $T(t)$ may be expressed in terms of a path integral as follows: For $x, y \in \mathbb{R}^{3}$ and $t>0$, let $d \mu_{x, y, t}$ be the conditional Wiener measure on continuous paths $\beta:[0, t] \rightarrow \mathbb{R}^{3}$, such that $\beta_{0}=x, \beta_{t}=y$. Then

$$
T(t)(x, y)=H_{t}(x, y) \int \exp \left[-\int_{0}^{t} W\left(\beta_{s}\right) d s\right] d \mu_{x, y, t} .
$$

Hence $T(t)(x, y)$ is positive for all $x, y \in \mathbb{R}^{3}$ and one can see [1] that $T(t)(x, y)$ is continuous in $(x, y, t)$. Furthermore, it is evident that $T(t)(x, y)$ satisfies the inequality

$$
T(t)(x, y) \leqq H_{t}(x, y) \exp \left[\|W\|_{\infty} t\right], \quad t>0 .
$$

Here we wish to consider the operator $S=T-L_{\psi}$ on $L^{2}\left(\mathbb{R}^{3}\right)$, where $L_{\psi}$ is given by (2.2).

Lemma 3.1. The operator $S$ is essentially self-adjoint on $L^{2}\left(\mathbb{R}^{3}\right)$ and generates a strongly continuous semi-group $S(t), t>0$. The operators $S(t)$ may be written as an infinite series

$$
S(t)=\sum_{n=0}^{\infty} S_{n}(t)
$$


converging in the uniform norm, where $S_{0}(t)=T(t)$ and

$$
S_{n}(t)=\int_{0}^{t} T(t-\tau) L_{\psi} S_{n-1}(\tau) d \tau, \quad n \geqq 1 .
$$

Proof. Let $u \in L^{2} \cap L^{\infty}$. Then one can easily see from (2.2) that $L_{\psi} u \in L^{2}$, and there exist constants $a, b>0$ such that

$$
\left\|L_{\psi} u\right\|_{2} \leqq a\|u\|_{2}+b\|u\|_{\infty} .
$$

The essential self-adjointness of $S$ now follows from the fact that there exist constants $c, d>0$ with

$$
\|u\|_{\infty} \leqq c\|u\|_{2}+d\|\Delta u\|_{2},
$$

and the Kato-Rellich theorem [13]. Furthermore, the domains of the operators $S$, $T$, and $(-\Delta)$ are identical. Taking a function $g$ in any of these domains, we see from (3.6) that there is a constant $C$ such that

$$
\left\|L_{\psi} T(t) g\right\|_{2} \leqq \frac{c}{t^{3 / 4}}\|g\|_{2}, \quad 0<t<1 .
$$

Applying Theorem 8.1.19 of [5], we conclude that $S$ generates a strongly continuous semi-group $S(t)=\exp (-S t), t>0$, with the properties stated in the lemma.

Lemma 3.2. Each operator $S_{n}(t), n \geqq 1$, has a continuous kernel $S_{n}(t)(x, y)$ which is symmetric. There exists a universal constant $A$ such that for any $r, 2 \leqq r \leqq \infty$, and $y \in \mathbb{R}^{3}$, the kernel satisfies the inequality

$$
\left\|S_{n}(t)(\cdot, y)\right\|_{r} \leqq \frac{A^{n} \exp \left[\|W\|_{\infty} t\right]}{\Gamma(n / 2+1)}\|\psi\|_{2}^{2 n} t^{(n-3+3 / r) / 2}, \quad t>0 .
$$

Here $\Gamma$ denotes the Euler gamma function.

Proof. The symmetry of $S_{n}(t)$ follows from the representation (3.5) and the selfadjointness of $L_{\psi}$ and $T(t)$. The continuity of $S_{n}(t)(x, y)$ may be deduced from the following argument to obtain the bound (3.9).

Putting $M=\|W\|_{\infty}$, we have from (2.6) if $0<\tau<t$,

$$
\left|T(t-\tau) L_{\psi} S_{n-1}(\tau)(x, y)\right| \leqq e^{M(t-\tau)} C_{p}\left\|H_{t-\tau}(x, \cdot) \psi\right\|_{p}\left\|S_{n-1}(\tau)(\cdot, y) \psi\right\|_{q} .
$$

On applying Hölder's inequality, we have

$$
\left\|S_{n-1}(\tau)(\cdot, y) \psi\right\|_{q} \leqq\left\|S_{n-1}(\tau)(\cdot, y)\right\|_{s}\|\psi\|_{2},
$$

where $s$ is given by (2.7). From Young's inequality we see that for $2 \leqq r \leqq \infty$ the function $\left\|H_{t-\tau}(x, \cdot) \psi\right\|_{p} \in L^{r}\left(\mathbb{R}^{3}\right)$ and

$$
\|\| H_{t-\tau}(x, \cdot) \psi\left\|_{p}\right\|_{r} \leqq a[2 \pi(t-\tau)]^{-\eta_{r p}}\|\psi\|_{2},
$$

where $\eta_{r p}$ is given by

$$
\eta_{r p}=\frac{3}{2}\left[\frac{3}{2}-\frac{1}{r}-\frac{1}{p}\right]
$$


and $a$ is a constant independent of $r, p$ provided $1 \leqq p \leqq 3 / 2,2 \leqq r \leqq \infty$. If we use the fact that

$$
\left\|S_{0}(t)(\cdot, y)\right\|_{s} \leqq b e^{M t}(2 \pi t)^{-3(1-1 / s) / 2}, \quad 2 \leqq s \leqq \infty,
$$

where $b$ is a constant independent of $s$, we see from (3.5) and (3.10) that

$$
\left\|S_{1}(t)(\cdot, y)\right\|_{r} \leqq C_{p} a b e^{M t}\|\psi\|_{2}^{2} \int_{0}^{t}[2 \pi(t-\tau)]^{-\eta_{r p}}(2 \pi \tau)^{-3(1-1 / s) / 2} d \tau .
$$

Since $p$ and $s$ in (3.15) can be chosen arbitrarily subject to the conditions $p^{-1}+s^{-1}=7 / 6,1<p<3 / 2$, we find that for some constant $A$ the following inequality holds:

$$
\left\|S_{1}(t)(\cdot, y)\right\|_{r} \leqq A e^{M t}\|\psi\|_{2}^{2} t^{(-1+3 / 2 r)}, \quad 2 \leqq r \leqq \infty .
$$

The inequality (3.9) now easily follows by an induction argument.

Next for $-\infty<\lambda<\infty$, let $P(\lambda)$ be the projection of the self-adjoint operator $S$ on the interval $(-\infty, \lambda]$. By $(2.5)$ we see that $S$ is bounded below, and hence there exists $\lambda_{0} \leqq 0$ such that $P(\lambda)=0$ if $\lambda<\lambda_{0}$.

Lemma 3.3. For every $\lambda \in \mathbb{R}, P(\lambda)$ is an integral operator with continuous kernel $P(\lambda)(x, y)$. For each $x \in \mathbb{R}^{3}, P(\lambda)(x, x)$ generates a positive Borel measure $d P(\lambda)(x, x)$ such that

$$
S(t)(x, x)=\int_{\lambda_{0}}^{\infty} e^{-\lambda t} d P(\lambda)(x, x), \quad t>0 .
$$

Proof. From Lemmas 3.1 and 3.2 we conclude that $S(t)$ is an integral operator with continuous kernel $S(t)(x, y)$. Let $Q(\lambda)$ be the operator $Q(\lambda)=q(S)$, where $q:(-\infty, \infty) \rightarrow \mathbb{R}$ is given by $q(z)=0$ if $z>\lambda, q(z)=e^{2 z}$ if $z \leqq \lambda$. Thus we have

$$
P(\lambda)=S(1) Q(\lambda) S(1) \text {. }
$$

Note from the proof of Lemma 3.2 that $S(1)$ may be regarded as a continuous mapping $h: \mathbb{R}^{3} \rightarrow L^{2}\left(\mathbb{R}^{3}\right)$ by setting

$$
h(y)=S(1)(\cdot, y), \quad y \in \mathbb{R}^{3} .
$$

Thus for $u \in L^{2}\left(\mathbb{R}^{3}\right)$ with bounded support, it follows that

$$
S(1) u=\int_{\mathbb{R}^{3}} h(y) u(y) d y .
$$

Applying $Q(\lambda)$ to both sides of (3.20) we obtain

$$
Q(\lambda) S(1) u=\int_{\mathbb{R}^{3}} Q(\lambda) h(y) u(y) d y,
$$

in view of the fact that $Q(\lambda)$ is a bounded operator. Again from Lemma 3.2 we see that $S(1)$ is a bounded operator from $L^{2}\left(\mathbb{R}^{3}\right)$ to $C_{0}\left(\mathbb{R}^{3}\right)$. It therefore follows from (3.18) and (3.21) that $P(\lambda)$ is an integral operator with continuous kernel

$$
P(\lambda)(x, y)=S(1)[Q(\lambda) h(y)](x) .
$$


To show that $d P(\lambda)(x, x)$ is a positive measure, we note that for $\lambda>\lambda^{1}$ the operator $P(\lambda)-P\left(\lambda^{1}\right)$ is positive and so we may conclude that

$$
P(\lambda)(x, x) \geqq P\left(\lambda^{1}\right)(x, x)
$$

for any $x \in \mathbb{R}^{3}$. Hence $P(\lambda)(x, x)$ is an increasing function of $\lambda$ and from (3.22) we see that this function is continuous from above, so we may form the Borel measure $d P(\lambda)(x, x)$ on $(-\infty, \infty)$. The identity (3.17) now follows from the fact that for any $u \in L^{2}$,

$$
(S(t) u, u)=\int_{\lambda_{0}}^{\infty} e^{-\lambda t} d(P(\lambda) u, u)
$$

by choosing functions $u$ which converge to the Dirac $\delta$ function at $x$.

Lemma 3.4. Let $\mathscr{D} \subset \mathbb{R}^{3}$ be a bounded domain, and associate with $\mathscr{D}$ a measure $\mu_{\mathscr{D}}$ on $\mathbb{R}^{2}$ defined by

$$
\mu_{\mathscr{D}}\{(-\infty, \lambda] x(-\infty, v]\}=\int_{\mathscr{D}} \int_{\mathscr{D}} \frac{P(\lambda)(x, y) P(v)(y, x)}{|x-y|} d x d y .
$$

Then $\mu_{\mathscr{D}}$ is a positive Borel measure on $\mathbb{R}^{2}$ with support in the set $\left\{(\lambda, v): \lambda \geqq \lambda_{0}\right.$, $\left.v \geqq \lambda_{0}\right\}$, and for any $t, \tau>0$, the following identity holds:

$$
\int_{\mathscr{D}} \int_{\mathscr{D}} \frac{S(t)(x, y) S(\tau)(y, x)}{|x-y|} d x d y=\int_{\lambda_{0}}^{\infty} \int_{\lambda_{0}}^{\infty} e^{-(\lambda t+v \tau)} d \mu_{\mathscr{D}}(\lambda, v) .
$$

Proof. We first show that the right side of (3.24) is positive. We consider $P(\lambda)$ restricted to $L^{2}(\mathscr{D})$. Then $P(\lambda)$ has eigenvalues $a_{1} \geqq a_{2} \geqq \ldots \geqq 0$ with corresponding eigenfunctions $\psi_{i}(x), i \geqq 1$. The functions $\psi_{i}(x)$ are continuous on the closure of $\mathscr{D}$ and

$$
P(\lambda)(x, y)=\sum_{i=1}^{\infty} a_{i} \psi_{i}(x) \overline{\psi_{i}(y)}, \quad x, y \in \mathscr{D},
$$

where the series in (3.26) converges in $L^{2}(\mathscr{D} \times \mathscr{D})$. We have a similar expression for $P(v)(x, y)$, namely

$$
P(v)(x, y)=\sum_{j=1}^{\infty} b_{j} \phi_{j}(x) \overline{\phi_{j}(y)}, \quad x, y \in \mathscr{D} .
$$

Let $P_{i}(\lambda)(x, y), P_{j}(v)(x, y)$ denote the $i^{\text {th }}$ and $j^{\text {th }}$ partial sums in (3.26) and (3.27), respectively. Then since $|x|^{-1}$ is positive definite, the integral on the right in (3.24) is positive if we replace $P(\lambda)(x, y)$ by $P_{i}(\lambda)(x, y)$ and $P(v)(x, y)$ by $P_{j}(v)(x, y)$. Now, using the fact that

$$
P(\lambda)(x, x)=\sum_{i=1}^{\infty} a_{i}\left|\psi_{i}(x)\right|^{2}, \quad x \in \mathscr{D}
$$

where the convergence in (3.28) is pointwise, we conclude from the dominated convergence theorem that the right side of (3.24) is positive. It follows by a similar argument that $\mu_{\mathscr{D}}$ is a finitely additive positive measure on $\mathbb{R}^{2}$. From (3.18) we see that $\mu_{\mathscr{D}}$ is continuous from above and hence a Borel measure on $\mathbb{R}^{2}$. 
To prove (3.25) we observe that the function $P(\lambda)(x, y)$ of $\lambda$ generates a signed measure $d P(\lambda)(x, y)$ on $\mathbb{R}$ with total variation measure $|d P(\lambda)(x, y)|$ satisfying

$$
|d P(\lambda)(x, y)| \leqq \frac{1}{2}[d P(\lambda)(x, x)+d P(\lambda)(y, y)] .
$$

Hence by (3.17) the Laplace transform of $d P(\lambda)(x, y)$ exists, and one can easily see it is given by

$$
S(t)(x, y)=\int_{\lambda_{0}}^{\infty} e^{-\lambda t} d P(\lambda)(x, y), \quad t>0 .
$$

The formula (3.25) now follows from (3.30) by using Fubini's theorem and the fact that $d P(\lambda)(x, y) d P(v)(y, x) d x d y$ is a signed measure on $\mathbb{R}^{2} \times \mathbb{R}^{3} \times \mathbb{R}^{3}$.

We introduce a parameter $\gamma>0$ and define the operator $T^{\gamma}=H+\gamma W$ with corresponding semi-group $T^{\gamma}(t)$ and the operator $S^{\gamma}=T^{\gamma}-L_{\psi}$ with semi-group $S^{\gamma}(t)$.

Lemma 3.5. The following limits exist as $\gamma \rightarrow \infty$ : for any $m>0, x \in \mathbb{R}^{3}$,

$$
\lim _{\gamma \rightarrow \infty} \gamma^{-3 / 2} S^{\gamma}(m / \gamma)(x, x)=(2 \pi m)^{-3 / 2} \exp [-m W(x)] .
$$

For any $m, n>0$, and bounded domain $\mathscr{D} \subset \mathbb{R}^{3}$,

$$
\begin{aligned}
& \lim _{\gamma \rightarrow \infty} \gamma^{-2} \int_{\mathscr{D}} \int_{\mathscr{D}} \frac{S^{\gamma}(m / \gamma)(x, y) S^{\gamma}(n / \gamma)(y, x)}{|x-y|} d x d y \\
& \quad=(2 \pi)^{-2}(m n)^{-1 / 2}(m+n)^{-1} \int_{\mathscr{D}} \exp [-(m+n) W(x)] d x .
\end{aligned}
$$

Proof. By Lemma 3.2 we see that

$$
\left|S^{\gamma}(m / \gamma)(x, x)-S_{0}^{\gamma}(m / \gamma)(x, x)\right|=O(\gamma),
$$

as $\gamma \rightarrow \infty$. Thus it is sufficient to prove (3.31) with $S^{\gamma}$ replaced by $S_{0}^{\gamma}=T^{\gamma}$. From (3.2) this is equivalent to showing that

$$
\lim _{\gamma \rightarrow \infty} \int \exp \left[-\gamma \int_{0}^{m / \gamma} W\left(\beta_{s}\right) d s\right] d \mu_{x, x, m / \gamma}=\exp [-m W(x)] .
$$

Let $b(s), s \geqq 0$, be Brownian motion in $\mathbb{R}^{3}$ starting at 0 . Then we have [15] for the $\beta_{s}$ of (3.34) the relation

For $\delta>0$, let

$$
\beta_{s}-x=b(s)-\frac{s \gamma}{m} b(m / \gamma), \quad 0 \leqq s \leqq m / \gamma .
$$

$$
N_{\delta}=\left\{\beta: \sup _{0 \leqq s \leqq m / \gamma}\left|\beta_{s}-x\right| \leqq \delta\right\}, \quad \text { and } \sim N_{\delta} \text { be the complement of } N_{\delta} .
$$

Then from (3.35) and the reflection principle for Brownian motion we see that there is a constant $c>0$ such that as $\gamma \rightarrow \infty$,

$$
\int_{\sim N_{\delta}} d \mu_{x, x, m / \gamma} \leqq e^{-c \gamma}
$$


We write the integral on the left in (3.34) as a sum of integrals over $N_{\delta}$ and $\sim N_{\delta}$. By (3.37) we have

$$
\int_{\sim N_{\delta}} \exp \left[-\gamma \int_{0}^{m / \gamma} W\left(\beta_{s}\right) d s\right] d \mu_{x, x, m / \gamma} \leqq \exp \left[m\|W\|_{\infty}-c \gamma\right],
$$

which converges to zero as $\gamma \rightarrow \infty$. Hence by letting $\delta \rightarrow 0$ and using the continuity of $W$ at $x$, we obtain (3.34). We have therefore proved (3.31).

We turn to (3.32). As for (3.31) we first show that it is sufficient to prove (3.32) with $S^{\gamma}$ replaced by $S_{0}^{\gamma}$. By Lemma 3.2 there is a constant $C$ such that the following inequalities hold as $\gamma \rightarrow \infty$ for any $x, y \in \mathbb{R}^{3}, 2 \leqq r \leqq \infty$ :

$$
\begin{array}{r}
\left\|S^{\gamma}\left(\frac{m}{\gamma}\right)(x, \cdot)\right\|_{r} \leqq C \gamma^{3(1-r) / 2}, \\
\left\|S^{\gamma}\left(\frac{m}{\gamma}\right)(x, \cdot)-S_{0}^{\gamma}\left(\frac{m}{\gamma}\right)(x, \cdot)\right\|_{r} \leqq C \gamma^{1-3 / 2 r} .
\end{array}
$$

Hence we have

$$
\begin{aligned}
& \int_{\mathscr{D}} \int_{\mathscr{D}} \frac{\left|S^{\gamma}(m / \gamma)(x, y)\right|\left|S^{\gamma}(n / \gamma)(y, x)-S_{0}^{\gamma}(n / \gamma)(y ; x)\right|}{|x-y|} d x d y \\
& \quad \leqq C \gamma^{3 / 2} \int_{\mathscr{D}} d y \int_{\mathscr{D}} \frac{\left|S^{\gamma}(n / \gamma)(y, x)-S_{0}^{\gamma}(n / \gamma)(y, x)\right|}{|x-y|} d x \\
& \quad \leqq C \gamma^{3 / 2} \int_{\mathscr{D}} d y\left[\int_{\mathscr{D}} \frac{d x}{|x-y|^{2}}\right]^{1 / 2}\left\|S^{\gamma}(n / \gamma)(y, \cdot)-S_{0}^{\gamma}(n / \gamma)(y, \cdot)\right\|_{2} \\
& \quad \leqq C^{2} \gamma^{7 / 4} \int_{\mathscr{D}} d y\left[\int_{\mathscr{D}} \frac{d x}{|x-y|^{2}}\right]^{1 / 2},
\end{aligned}
$$

from (3.39), (3.40), and the Hölder inequality. Since $7 / 4<2,(3.41)$ shows that it is sufficient for us to prove (3.32) for $S_{0}^{\gamma}$.

From (3.2) we have

$$
S_{0}^{\gamma}(m / \gamma)(x, y)=H_{m / \gamma}(x, y) \int \exp \left[-\gamma \int_{0}^{m / \gamma} W\left(\beta_{s}\right) d s\right] d \mu_{x, y, m / \gamma} .
$$

It easily follows from (3.42) that for any $\delta>0$, there is a $c>0$ such that $S_{0}^{\gamma}(m / \gamma)(x, y)$ tends to zero faster than $\exp (-c \gamma)$ as $\gamma \rightarrow \infty$ in the set $\{(x, y):|x-y|>\delta / 2\}$. It is therefore sufficient to prove (3.32) with $S^{\gamma}$ replaced by $S_{0}^{\gamma}$ and the domain of integration $\mathscr{D} \times \mathscr{D}$ replaced by $\{(x, y) \in \mathscr{D} \times \mathscr{D}:|x-y|<\delta / 2\}$. The process $\beta_{\text {s }}$ in (3.42) is related to the standard Brownian motion $b(s)$ by

$$
\beta_{s}-x=\frac{\gamma s}{m}(y-x)+b(s)-\frac{s \gamma}{m} b\left(\frac{m}{\gamma}\right), \quad 0 \leqq s \leqq m / \gamma .
$$

Hence we see just as in (3.37) that if $|x-y|<\delta / 2$, then there is a constant $c>0$ such that as $\gamma \rightarrow \infty$,

$$
\int_{\sim N_{\delta}} d \mu_{x, y, m / \gamma} \leqq e^{-c \gamma}
$$


It follows that in evaluating (3.32) we may replace $S_{0}^{\gamma}(m / \gamma)(x, y)$ by

$$
H_{m / \gamma}(x, y) \int_{\sim N_{\delta}} \exp \left[-\gamma \int_{0}^{m / \gamma} W\left(\beta_{s}\right) d s\right] d \mu_{x, y, m / \gamma} .
$$

The asymptotic formula (3.32) now easily follows by letting $\delta \rightarrow 0$ in (3.45) and using the fact that $W(x)$ is uniformly continuous in $\mathscr{D}$. This completes the proof.

We let $P^{\gamma}(\lambda),-\infty<\lambda<\infty$, be the projection operators associated with $S^{\gamma}$. By (2.5) we see that there is a $\lambda_{0} \leqq 0$ independent of $\gamma$ such that $P^{\gamma}(\lambda)=0$ if $\lambda<\gamma \lambda_{0}$. In the following we denote by $f_{+}$the positive part of a function $f: \mathbb{R}^{3} \rightarrow \mathbb{R}$.

Thus

$$
f_{+}(x)=\max \{f(x), 0\}
$$

Theorem 3.6. The following asymptotic formula holds:

$$
\lim _{\gamma \rightarrow \infty} \gamma^{-3 / 2} P^{\gamma}(0)(x, x)=\frac{1}{6 \pi^{2}}[-W(x)]_{+}^{3 / 2}, \text { for any } x \in \mathbb{R}^{3} \text {. }
$$

Proof. Observe that the right side of (3.31) may be written as a Laplace transform,

$$
\frac{1}{4 \pi^{2}} \int_{\lambda_{0}}^{\infty} e^{-m u}[u-W(x)]_{+}^{1 / 2} d u
$$

where $\lambda_{0} \leqq-\|W\|_{\infty}$. The left side of (3.31) may also be written as a Laplace transform

$$
\int_{\lambda_{0}}^{\infty} e^{-m u} \gamma^{-3 / 2} d P^{\gamma}(\gamma u)(x, x)
$$

The result (3.47) now follows from the Tauberian theorem of Karamata [15] which we state in the form:

Lemma 3.7. Let $m^{\gamma}, 0<\gamma \leqq \infty$, be a set of positive Borel measures on the interval $\left[\lambda_{0}, \infty\right)$, such that for every $\alpha>0,0<\gamma \leqq \infty$,

$$
\int_{\lambda_{0}}^{\infty} e^{-\alpha u} d m^{\gamma}(u)<\infty,
$$

and

$$
\lim _{\gamma \rightarrow \infty} \int_{\lambda_{0}}^{\infty} e^{-\alpha u} d m^{\gamma}(u)=\int_{\lambda_{0}}^{\infty} e^{-\alpha u} d m^{\infty}(u) .
$$

Then the following asymptotic formula holds:

$$
\lim _{\gamma \rightarrow \infty} m^{\gamma}\left[\lambda_{0}, 0\right]=m^{\infty}\left[\lambda_{0}, 0\right] \text {. }
$$

Next let $\mu_{\mathscr{D}}^{\gamma}$ be the measure (3.24) associated with $P^{\gamma}(\lambda)$. Then

Theorem 3.8. The following asymptotic formula holds:

$$
\lim _{\gamma \rightarrow \infty} \gamma^{-2} \mu_{\mathscr{D}}^{\gamma}\{(-\infty, 0] x(-\infty, 0]\}=\frac{1}{2 \pi^{3}} \int_{\mathscr{D}}[-W(x)]_{+}^{2} d x .
$$


Proof. Let $f(u, v)$ be defined for $u, v \geqq 0$ by

$$
f(u, v)=\frac{1}{2 \pi^{3}} \log \left[\frac{u^{1 / 2}+v^{1 / 2}}{|u-v|^{1 / 2}}\right],
$$

and $b(u, v)$ be defined for $(u, v) \in \mathbb{R}^{2}$ by

$$
b(u, v)=\int_{\mathscr{D}} f\left([u-W(x)]_{+},[v-W(x)]_{+}\right) d x .
$$

Then one can see [2] that the right side of (3.32) is a two dimensional Laplace transform,

$$
\int_{\lambda_{0}}^{\infty} \int_{\lambda_{0}}^{\infty} e^{-(m u+n v)} b(u, v) d u d v
$$

From (3.25) we see that the left side of (3.32) is also a Laplace transform,

$$
\int_{\lambda_{0}}^{\infty} \int_{\lambda_{0}}^{\infty} e^{-(m u+n v)} \gamma^{-2} d \mu_{\mathscr{D}}^{\gamma}(\gamma u, \gamma v)
$$

The theorem now follows from a two dimensional version of Lemma 3.7:

Lemma 3.9. Let $m^{\gamma}, 0<\gamma \leqq \infty$, be a set of positive Borel measures on the subset $\left[\lambda_{0}, \infty\right) x\left[\lambda_{0}, \infty\right)$ of $\mathbb{R}^{2}$, such that for all $\alpha, \beta>0,0<\gamma \leqq \infty$,

$$
\int_{\lambda_{0}}^{\infty} \int_{\lambda_{0}}^{\infty} e^{-(\alpha u+\beta v)} d m^{\gamma}(u, v)<\infty
$$

and

$$
\lim _{\gamma \rightarrow \infty} \int_{\lambda_{0}}^{\infty} \int_{\lambda_{0}}^{\infty} e^{-(\alpha u+\beta v)} d m^{\gamma}(u, v)=\int_{\lambda_{0}}^{\infty} \int_{\lambda_{0}}^{\infty} e^{-(\alpha u+\beta v)} d m^{\infty}(u, v) .
$$

Then the following asymptotic formula holds:

$$
\lim _{\gamma \rightarrow \infty} m^{\gamma}\left\{\left[\lambda_{0}, 0\right] x\left[\lambda_{0}, 0\right]\right\}=m^{\infty}\left\{\left[\lambda_{0}, 0\right] x\left[\lambda_{0}, 0\right]\right\} .
$$

Remark. The techniques of this section are limited to potentials $W \in L^{\infty}$. One would clearly like to extend the results to potentials $W$ in $L^{p}+L^{\infty}$ for $p>3 / 2$. We can show, using a bound of Carmona [1], that (3.34) holds for such potentials if $p>3$. By considering the Coulomb potential, one can see that (3.34) does not necessarily hold if $p<3$. Thus to prove (3.47) and (3.53) for potentials in $L^{p}+L^{\infty}$ with $p>3 / 2$ one requires a technique entirely different from the one presented here.

\section{Hartree-Fock Theory}

We use the notation of Sect. 1. With $\lambda$ satisfying (1.19) and $\alpha>0$ such that $\alpha \lambda$ is an integer, let ${ }_{\alpha} \psi_{1}, \ldots,{ }_{\alpha} \psi_{\alpha \lambda}$ be the orthonormal set of wave functions representing a Hartree-Fock ground state for the Hamiltonian $H_{\alpha \lambda}\left(V_{\alpha}\right)$. Then ${ }_{\alpha} \psi_{1}, \ldots,{ }_{\alpha} \psi_{\alpha \lambda}$ are the first $\alpha \lambda$ eigenfunctions of the operator $S\left({ }_{\alpha} \psi_{1}, \ldots,{ }_{\alpha} \psi_{\alpha \lambda}\right)$ in (1.14), where we replace $V$ in the expression for $\phi_{\varrho}$ by $V_{\alpha}$. Let $L^{\alpha}$ be the nonlocal potential defined by

$$
L^{\alpha}=\alpha^{-4 / 3} L\left({ }_{\alpha} \psi_{1}\left(\alpha^{-1 / 3} \cdot\right), \ldots,{ }_{\alpha} \psi_{\alpha \lambda}\left(\alpha^{-1 / 3} \cdot\right)\right),
$$


where the ${ }_{\alpha} \psi_{i}\left(\alpha^{-1 / 3} \cdot\right)$ denote the functions ${ }_{\alpha} \psi_{i}\left(\alpha^{-1 / 3} x\right)$ and $L$ is given by (1.13). We put

$$
S^{\alpha}=-h^{2}\left(8 \pi^{2} m\right)^{-1} \Delta-\alpha^{-2 / 3} \phi_{\varrho_{\alpha}}\left(\alpha^{-1 / 3} x\right)-L^{\alpha} .
$$

Then it is clear that ${ }_{\alpha} \psi_{i}\left(\alpha^{-1 / 3} x\right), 1 \leqq i \leqq \alpha \lambda$, are the first $\alpha \lambda$ eigenfunctions of $S^{\alpha}$.

We need to strengthen (1.29). Let $\Phi_{\alpha}(x)$ be given by

$$
\Phi_{\alpha}(x)=\alpha^{-4 / 3} \phi_{\varrho_{\alpha}}\left(\alpha^{-1 / 3} x\right)-V(x) .
$$

Lemma 4.1. For $\alpha>0$ the function $\Phi_{\alpha}(x)$ is in $L^{\infty}\left(\mathbb{R}^{3}\right)$ and $\left\|\Phi_{\alpha}\right\|_{\infty}$ is bounded as $\alpha \rightarrow \infty$. The functions $\Phi_{\alpha}$ converge uniformly on compact sets as $\alpha \rightarrow \infty$ to the function $\phi_{\lambda}^{\mathrm{TF}}(x)-V(x)$.

Proof. We have

$$
\Phi_{\alpha}(x)=\alpha^{-4 / 3} \int_{\Omega} \frac{\varrho_{\alpha}(y)}{\left|\alpha^{-1 / 3} x-y\right|} d y
$$

We can write the integral in (4.4) as a sum

$$
\Phi_{\alpha}(x)=\alpha^{-4 / 3} \int_{\left|y-\alpha^{-1 / 3} x\right|>\alpha^{-1 / 3}}+\alpha^{-4 / 3} \int_{\left|y-\alpha^{-1 / 3} x\right| \leqq \alpha^{-1 / 3}} .
$$

The first term in (4.5) may be estimated as

$$
\alpha^{-4 / 3} \int_{\left|y-\alpha^{-1 / 3} x\right|>\alpha^{-1 / 3}} \leqq \alpha^{-1} \int_{\Omega} \varrho_{\alpha}(y) d y=\lambda .
$$

To estimate the second term we apply Hölder's inequality. Thus

$$
\alpha^{-4 / 3} \int_{\left|y-\alpha^{-1 / 3} x\right| \leqq \alpha^{-1 / 3}} \leqq(8 \pi)^{2 / 5} \alpha^{-7 / 5}\left[\int_{\Omega} \varrho_{\alpha}(y)^{5 / 3} d y\right]^{3 / 5} .
$$

Now we use Lieb's lower bound on the kinetic energy of fermions [6]. Thus there is a universal constant $C$ such that

$$
\int_{\Omega} \varrho_{\alpha}(y)^{5 / 3} d y \leqq C K\left({ }_{\alpha} \psi_{1}, \ldots,{ }_{\alpha} \psi_{\alpha \lambda}\right)
$$

Hence from (1.23) we see that the right side of (4.7) is bounded as $\alpha \rightarrow \infty$. It follows that $\left\|\Phi_{\alpha}\right\|_{\infty}$ is bounded as $\alpha \rightarrow \infty$.

For $0<\sigma<1$ and $x \neq z$ we consider

$$
\frac{\left|\Phi_{\alpha}(x)-\Phi_{\alpha}(z)\right|}{|x-z|^{\sigma}} \leqq \alpha^{-4 / 3} \int_{\Omega}\left|\frac{1}{\left|\alpha^{-1 / 3} x-y\right|}-\frac{1}{\left|\alpha^{-1 / 3} z-y\right|}\right| \frac{\varrho_{\alpha}(y) d y}{|x-z|^{\sigma}} .
$$

We estimate the integral in (4.9) to obtain

$$
\frac{\left|\Phi_{\alpha}(x)-\Phi_{\alpha}(z)\right|}{|x-z|^{\sigma}} \leqq \alpha^{-5 / 3} \int_{\Omega} \frac{|x-z|^{1-\sigma}}{\left|\alpha^{-1 / 3} x-y\right|\left|\alpha^{-1 / 3} z-y\right|} \varrho_{\alpha}(y) d y .
$$

We write the right side of (4.10) as a sum of three integrals $I_{1}+I_{2}+I_{3}$, where

$$
\begin{aligned}
& I_{1}=\alpha^{-5 / 3} \int_{\left|\alpha^{-1 / 3} x-y\right|<2^{-1} \alpha^{-1 / 3}|x-z|}, \\
& I_{2}=\alpha^{-5 / 3} \int_{\left|\alpha^{-1 / 3} z-y\right|<2^{-1} \alpha^{-1 / 3}|x-z|}, \\
& I_{3}=\alpha^{-5 / 3} \quad \int_{\inf \left[\left|\alpha^{-1 / 3} x-y\right|,\left|\alpha^{-1 / 3} z-y\right|\right]>2^{-1} \alpha^{-1 / 3}|x-z|} .
\end{aligned}
$$


Evidently we have

$$
I_{1} \leqq 2^{1-\sigma} \alpha^{-4 / 3-\sigma / 3} \int_{\Omega} \frac{\varrho_{\alpha}(y)}{\left|\alpha^{-1 / 3} x-y\right|^{1+\sigma}} d y .
$$

The integral in (4.14) may be estimated just the way we estimated (4.4). We conclude that if $\sigma<1 / 5$, then $I_{1}$ is bounded as $\alpha \rightarrow \infty$. To estimate $I_{3}$ we observe that for $y$ in the set $\left\{y: \inf \left[\left|\alpha^{-1 / 3} x-y\right|,\left|\alpha^{-1 / 3} z-y\right|\right]>2^{-1} \alpha^{-1 / 3}|x-z|\right\}$,

$$
\left|\alpha^{-1 / 3} x-y\right| \leqq 3\left|\alpha^{-1 / 3} z-y\right|
$$

and so we have

$$
I_{3} \leqq 3.2^{1-\sigma} \alpha^{-4 / 3-\sigma / 3} \int_{\Omega} \frac{\varrho_{\alpha}(y)}{\left|\alpha^{-1 / 3} x-y\right|^{1+\sigma}} d y,
$$

which shows that $I_{3}$ is bounded as $\alpha \rightarrow \infty$.

Thus the left side of (4.11) is uniformly bounded in $x$ and $z$ as $\alpha \rightarrow \infty$ provided $\sigma<1 / 5$. Hence the functions $\Phi_{\alpha}$ are equicontinuous on any compact set. From (1.29) we know that the $\Phi_{\alpha}$ converge pointwise to the function $\phi_{\lambda}^{\mathrm{TF}}(x)-V(x)$ as $\alpha \rightarrow \infty$. Hence by the Arzela-Ascoli theorem we deduce that the $\Phi_{\alpha}$ converge uniformly on compact sets to this same function. This completes the proof of the lemma.

Next let $\varepsilon^{\alpha}$ be the $\alpha \lambda^{\text {th }}$ eigenvalue of the operator $S^{\alpha}$ in (4.2).

Lemma 4.2. $\lim _{\alpha \rightarrow \infty} \alpha^{-2 / 3} \varepsilon^{\alpha}=-\phi_{\lambda}$, where $\phi_{\lambda}$ is defined by (1.20).

Proof. We bound $S^{\alpha}$ above and below by simpler operators. Let $S_{0}^{\alpha}=S^{\alpha}+L^{\alpha}$, so $S_{0}^{\alpha}$ does not contain a nonlocal potential. Since $L^{\alpha}$ is positive definite, it follows that $S^{\alpha} \leqq S_{0}^{\alpha}$. To bound $S^{\alpha}$ from below we observe from Lemma 2.2 that

$$
\left(u, L^{\alpha} u\right) \leqq C \lambda^{1 / 2} \alpha^{1 / 6}\|u\|_{2}\|\nabla u\|_{2} .
$$

Hence if we define $S_{1}^{\alpha}$ by

$$
S_{1}^{\alpha}=-\left[h^{2}\left(8 \pi^{2} m\right)^{-1}-C \lambda^{1 / 2} \alpha^{-1 / 6} 2^{-1}\right] \Delta-\alpha^{-2 / 3} \phi_{\varrho_{\alpha}}\left(\alpha^{-1 / 3} x\right)-C \lambda^{1 / 2} \alpha^{1 / 2} 2^{-1},
$$

it is clear from (4.17) that $S_{1}^{\alpha} \leqq S^{\alpha}$.

For $\delta>0$, let $N_{\delta}\left(S^{\alpha}\right)$ be the number of bound states of $S^{\alpha}$ with eigenvalues less than $-\alpha^{2 / 3} \delta$. From the above we have

$$
N_{\delta}\left(S_{0}^{\alpha}\right) \leqq N_{\delta}\left(S^{\alpha}\right) \leqq N_{\delta}\left(S_{1}^{\alpha}\right),
$$

provided $\alpha$ is sufficiently large. Now we use the fact that $V(x)$ falls off at $\infty$ and Lemma 4.1 to conclude that

$$
\lim _{\alpha \rightarrow \infty} \alpha^{-1} N_{\delta}\left(S_{i}^{\alpha}\right)=c^{-3 / 2} \int_{\mathbb{R}^{3}}\left[\phi_{\mathrm{TF}}(x)-\delta\right]_{+}^{3 / 2} d x, \quad i=0,1,
$$

where $c$ is given by (1.17). This follows from the method of Dirichlet-Neumann bracketing [14]. From (4.19) we see that (4.20) also holds for the operator $S^{\alpha}$. Suppose $0<\lambda \leqq z$. Then from $(1.20)$ we see that if $\delta>\phi_{\lambda}$, then $\lim _{\alpha \rightarrow \infty} \alpha^{-1} N_{\delta}\left(S^{\alpha}\right)<\lambda$.
Consequently we conclude that

$$
\liminf _{\alpha \rightarrow \infty} \alpha^{-2 / 3} \varepsilon^{\alpha} \geqq-\delta
$$


If $\lambda<z$ and $\delta<\phi_{\lambda}$, then $\lim _{\alpha \rightarrow \infty} \alpha^{-1} N_{\delta}\left(S^{\alpha}\right)>\lambda$. Hence

$$
\limsup _{\alpha \rightarrow \infty} \alpha^{-2 / 3} \varepsilon^{\alpha} \leqq-\delta .
$$

Letting $\delta \rightarrow \phi_{\lambda}$ in (4.21) and (4.22) we obtain the result of the lemma in the case $\lambda<z$. For $\lambda=z$ we may take $\delta=0$ in (4.22), since $\varepsilon^{\alpha}<0$ for all $\alpha$. Then (4.21) implies the result.

Theorem 4.3. The asymptotic formula (1.28) holds for $x \in \mathbb{R}^{3}$.

Proof. We proceed in exactly the same way we obtained (3.47). First we prove the analogue of (3.31) for $S^{\alpha}$, taking $\gamma=\alpha^{2 / 3}$. By Lemma 4.1 the analogue of (3.34) holds for $S^{\alpha}$. From Lemma 2.2 we have

$$
\left|\left(v, L^{\alpha} u\right)\right| \leqq C_{r} \lambda^{1 / 2} \alpha^{1 / 6}\|u\|_{r}\|v\|_{s} .
$$

Thus for $2 \leqq r \leqq \infty$ there is a constant $C$ such that

$$
\left\|S^{\alpha}\left(\frac{m}{\alpha^{2 / 3}}\right)(x, \cdot)-S_{0}^{\alpha}\left(\frac{m}{\alpha^{2 / 3}}\right)(x, \cdot)\right\|_{r} \leqq C \alpha^{5 / 6-1 / r}, \quad \alpha \rightarrow \infty .
$$

It follows from (4.24) that (3.31) holds for $S^{\alpha}$. Let $P^{\alpha}(\xi)$ be the projection of the operator $S^{\alpha}$ on $(-\infty, \xi]$ for $-\infty<\xi<\infty$. Then we may obtain a version of (3.47) for $P^{\alpha}$ from the version of (3.31) for $S^{\alpha}$. We conclude that

$$
\lim _{\alpha \rightarrow \infty} \alpha^{-1} P^{\alpha}\left(\varepsilon^{\alpha}\right)(x, x)=\frac{1}{2} \varrho_{\lambda}^{\mathbf{T F}}(x), \quad x \in \Omega,
$$

by virtue of (1.20) and Lemma 4.2. The conclusion of the theorem follows immediately from (4.25).

Lemma 4.4. Let $\mathscr{D} \subset \mathbb{R}^{3}$ be a bounded domain and $\mathscr{D}_{1}=\mathscr{D} \times\{-1,1\} \subset \Omega$. Then

$$
\lim _{\alpha \rightarrow \infty} \alpha^{-4 / 3} \int_{\mathscr{D}_{1}} \int_{\mathscr{D}_{1}} \frac{1}{2} \frac{P^{\alpha}\left(\varepsilon^{\alpha}\right)(x, y) P^{\alpha}\left(\varepsilon^{\alpha}\right)(y, x)}{|x-y|} d x d y=3^{4 / 3} \pi^{-1 / 3} 4^{-1} \int_{\mathscr{D}} \varrho_{\lambda}^{\mathrm{TF}}(x)^{4 / 3} d x .
$$

Proof: First we observe that (3.32) holds with $S_{0}^{\alpha}$ in the place of $S^{\gamma}$ and $\gamma=\alpha^{2 / 3}$. This follows from Lemma 4.1. Then (3.32) with $S^{\alpha}$ in place of $S^{\gamma}$ is obtained from this by using (4.24). Finally we get (4.26) from Lemma 4.2 and (3.53).

Theorem 4.5. The asymptotic formula (1.27) holds.

Proof. Evidently (1.27) is (4.26) with $\mathscr{D}_{1}=\Omega$. Since we have proved (4.26) only for bounded domains $\mathscr{D}$, we need Lemma 2.3. Let $\mathscr{A} \subset \mathscr{D}$ be the set of $x \in \mathscr{D}$ whose distance to the complement of $\mathscr{D}, \sim \mathscr{D}$, is at least 1 . Then we have

$$
\left|\alpha^{-5 / 3} \operatorname{Ex}\left({ }_{\alpha} \psi_{1}, \ldots,{ }_{\alpha} \psi_{\alpha \lambda}\right)-\int_{\mathscr{D}_{1}} \int_{\mathscr{D}_{1}}\right| \leqq \int_{\sim \mathscr{A}_{1}} \int_{\sim \mathscr{A}_{1}}+2 \int_{\sim \mathscr{D}_{1}} \int_{\mathscr{A}_{1}}
$$

where the integrands in (4.27) are the same as in the left side of (4.26). Now by Lemma 2.3 we have

$$
\int_{\sim \mathscr{A}_{1}} \int_{\sim \mathscr{A}_{1}} \leqq C \alpha^{-4 / 3} \int_{\mathscr{A}}\left[\alpha^{-1} \varrho_{\alpha}\left(\alpha^{-1 / 3} x\right)\right]^{4 / 3} d x
$$


By Hölder's inequality we have

$$
\int_{\mathscr{A}} \varrho_{\alpha}\left(\alpha^{-1 / 3} x\right)^{4 / 3} d x \leqq\left[\int_{\mathscr{A}} \varrho_{\alpha}\left(\alpha^{-1 / 3} x\right) d x\right]^{1 / 2}\left[\int_{\mathscr{A}} \varrho_{\alpha}\left(\alpha^{-1 / 3} x\right)^{5 / 3} d x\right]^{1 / 2} .
$$

Thus, using Lieb's lower bound on the kinetic energy of fermions, we obtain

$$
\int_{\sim \mathscr{A}_{1}} \int_{\sim \mathscr{A}_{1}} \leqq C^{\prime}\left[\int_{\mathscr{A}} \alpha^{-2} \varrho_{\alpha}\left(\alpha^{-1 / 3} x\right) d x\right]^{1 / 2}, \quad \alpha \rightarrow \infty,
$$

for some constant $C^{\prime}$. The second integral on the right in (4.27) may be bounded as

$$
\begin{aligned}
2 \int_{\sim \mathscr{D}_{1}} \int_{\mathscr{A}_{1}} & \leqq \alpha^{-4 / 3} \int_{\Omega} \int_{\Omega} P^{\alpha}\left(\varepsilon^{\alpha}\right)(x, y) P^{\alpha}\left(\varepsilon^{\alpha}\right)(y, x) d x d y \\
& =\alpha^{-1 / 3} \lambda .
\end{aligned}
$$

Finally we let $\mathscr{D} \rtimes \mathbb{R}^{3}$. By the Lieb-Simon weak version of (1.28) the right side of (4.30) converges to zero. Letting $\alpha \rightarrow \infty$ we obtain the result of the theorem.

Acknowledgements. I wish to thank Rafael Benguria for teaching me about Thomas-Fermi theory. I wish also to thank Elliott Lieb for valuable discussions, especially for bringing my attention to his results in [9].

\section{References}

1. Carmona, R. : Regularity properties of Schrödinger and Dirchlet semi-groups. J. Functional Anal. 33, 259-296 (1976)

2. Conlon, J.: A refinement of the Thomas-Fermi approximation for the $N$ body problem. J. Math. Phys. (to appear)

3. Cwickel, M.: Weak type estimates for singular values and the number of bound states of Schrödinger operators. Ann. Math. 106, 93-100 (1977)

4. Dirac, P.: Note on exchange phenomena in the Thomas atom. Proc. Cambridge Philos. Soc. 26, 376-385 (1930)

5. Dunford, N., Schwartz, J.: Linear operators, Vol. I. New York: Interscience 1963

6. Lieb, E.: The stability of matter. Rev. Mod. Phys. 48, 553-569 (1976)

7. Lieb, E.: A lower bound for Coulomb energies. Phys. Lett. 70A, 444-446 (1979)

8. Lieb, E.: Thomas-Fermi and related theories of atoms and molecules. Rev. Mod. Phys. 53, 603-641 (1981)

9. Lieb, E.: An $L^{p}$ bound for the Reisz and Bessel potentials of orthonormal functions. Preprint 1982

10. Lieb, E., Oxford, S.: An improved lower bound on the indirect Coulomb energy. Int. J. Quant. Chem. 19, 427-439 (1981)

11. Lieb, E., Simon, B.: The Hartree-Fock theory for Coulomb systems. Commun. Math. Phys. 53, 185-194 (1977)

12. Lieb, E., Simon, B.: The Thomas-Fermi theory of atoms, molecules, and solids. Adv. Math. 23, 22-116 (1977)

13. Reed, M., Simon, B.: Methods of modern mathematical physics, Vol. II. New York: Academic Press 1975

14. Reed, M., Simon, B.: Methods of modern mathematical physics, Vol. IV. New York: Academic Press 1978

15. Simon, B.: Functional integration and quantum physics. New York: Academic Press 1979

Communicated by B. Simon

Received February 4, 1982; in revised form September 9, 1982 